\title{
B-Tensor: Brain Connectome Tensor Factorization for Alzheimer's Disease
}

\author{
Göktekin Durusoy, M.S., Zerrin Yıldırım, M.D., Demet Yüksel Dal, M.S., Cigdem Ulasoglu-Yildiz, Ph.D., \\ Elif Kurt, Ph.D., Güneş Bayır, M.S., Erhan Özacar, B.S., Evren Özarslan, Ph.D., Aslı Demirtaş-Tatlıdede, M.D., \\ Ph.D., Başar Bilgiç, M.D., Tamer Demiralp, M.D., Hakan Gurvit, M.D., Alkan Kabakçığlu, Ph.D., \\ and Burak Acar*, Ph.D., Senior Member, IEEE
}

\begin{abstract}
AD is the highly severe part of the dementia spectrum and impairs cognitive abilities of individuals, bringing economic, societal and psychological burdens beyond the diseased. A promising approach in AD research is the analysis of structural and functional brain connectomes, i.e. sNETs and fNETs, respectively. We propose to use tensor representation (B-tensor) of uni-modal and multi-modal brain connectomes to define a low-dimensional space via tensor factorization. We show on a cohort of 47 subjects, spanning the spectrum of dementia, that diagnosis with an accuracy of $77 \%$ to $100 \%$ is achievable in a 5D connectome space using different structural and functional connectome constructions in a uni-modal and multi-modal fashion. We further show that multi-modal tensor factorization improves the results suggesting complementary information in structure and function. A neurological assessment of the connectivity patterns identified largely agrees with prior knowledge, yet also suggests new associations that may play a role in the disease progress.
\end{abstract}

Index Terms-Brain Connectomes, Structure and Function, Tensor Factorization, Dementia, Alzheimer's Disease, fMRI, DTI

\section{INTRODUCTION}

$\mathbf{N}$ UERODEGENERATIVE diseases draw increasing attention among both industrial and academic researchers due to the increase in elderly population. As of 2019 , there were 703 million people in the world aged 65 and over, which is expected to double until 2050 , reaching $16 \%$ of the world population [1]. In parallel with this increase in the elderly population, the incidence of age-related neurodegenerative diseases, such as Alzheimer's Disease (AD), increases [2]-[4].

Though the cause and the mechanisms of dementia, most prominently the $\mathrm{AD}$, have not been fully understood, an in-

G. Durusoy, G. Bayır, D.Y. Dal and B. Acar are with VAVlab, Department of Electrical \& Electronics Engineering, Bogazici University, İstanbul, Turkey.

H. Gurvit, B. Bilgiç and A. Demirtaş-Tatlıdede are with Department of Neurology, Faculty of Medicine, Istanbul University, Çapa, İstanbul, Turkey.

E. Özarslan is with Department of Biomedical Engineering, Linköping University, Linköping, Sweden.

E. Özarslan and E. Özacar are with Dept. of Physics, Bogazici University, İstanbul, Turkey.

A. Demirtaş-Tatlıdede is with Department of Neurology, Faculty of Medicine, Bahcesehir University, İstanbul, Turkey.

C.U. Yıldız and T. Demiralp are with Hulusi Behçet Life Sciences Research Lab., Istanbul University, Çapa, İstanbul, Turkey.

Z. Yıldırım, E. Kurt are with Dept. of Neuroscience, Aziz Sancar Institute of Experimental Medicine, Istanbul University, Çapa, İstanbul, Turkey.

A. Kabakçığlu is with Dept. of Physics, Koc University, İstanbul, Turkey.

*Corresponding author (email: acarbu@boun.edu.tr)

Manuscript received XXX; revised XXX. creasingly promising approach is to describe it as a disconnectivity syndrome where connectivity between (cortical) brain regions are disrupted [5], [6]. Graph theory and graph-based data analysis offer a suitable approach and the necessary tools to study these disruptions. Brain connectome is a graph/network representation of the structural and functional associations between (cortical) brain regions [7]-[9]. The structural connectomes (sNET) and the functional connectomes (fNET) are constructed using co-registered T1-weighted MRI (T1w-MRI), diffusion weighted MRI (DWI) and functional MRI (fMRI) volumes.

Early graph theoretical efforts in brain connectome analysis mostly focused on studying the differences in graph-level features between groups [10]. Characteristic path length, smallworldness, clustering coefficient and global connectivity are among these features that are used to discriminate $\mathrm{AD}$ and controls [11]. Despite their relatively high sensitivity, they had low specificity and provided very limited insight about the mechanisms that govern the onset and progress of diseases [12]. Conflicting results were presented in literature [13]-[15]. Daianu et al. uses k-core measure with the other metrics mentioned above to highlight alterations as the disease progresses, and distinguish $\mathrm{AD}$ vs MCI (Mild Cognitive Impaired), and healthy controls [16]. Rather than using the whole brain graph, they prefer to use a sub-graph by using the constraint $k$ as a threshold of nodal degree. In another study, k-core measure is used with rich club to understand the effect of $\mathrm{AD}$ on the highly connected graphs [17]. In a recent review article, Dragomir et al. provides an overview of the graph based approaches applied to AD [18].

A major challenge in deciphering the dementia spectrum is in identifying the disrupted structural and/or functional associations throughout the disease spectrum. This is equivalent to identifying the subsets of structural and/or functional associations (i.e. sNET and/or fNET subnetworks) that have a discriminative power. Early work pursued a statistical approach. Network Based Statistics (NBS), proposed by Zalesky et al., identifies statistically significantly different associations and subnetworks between groups by means of hypothesis testing [19]. Kim et al. used individual edge weights to perform univariate general linear model in order to identify group differences between AD and healthy controls [20].

More recently, network factorization approaches have been applied to connectome analysis under different paradigms and with different formulations. Network factorization aims at 
representing a network as a combination of subnetworks that form a basis for the space of connectomes. The connectomes' representations with respect to such a basis were exploited to draw insights about neurodegenerative diseases. Eavani et al. sought for such basis subnetworks, termed as Sparse Connectivity Patterns (SCP), for resting state fNETs [21]. Using these basis networks, they have found functionally (anti-)correlated regions as represented by the reconstruction coefficients. Zille et al. studied the similarities and the differences in resting state fNETs between children and young adults [22]. They performed a coupled factorization on the complete cohort by using a generalized fused Lasso penalty, where SCPs were constrained to be common across the two groups. Durusoy et al., proposed the $3^{\text {rd }}$ order B-tensor (brain tensor) representation, which is a group-wide unified tensor representation of uni-modal brain connectomes, of a cohort of sNETs that they decomposed onto a set of (anatomically) predefined subnetworks and showed that among them the salience network [23] turns out to be the most discriminative one between controls and AD [24]. More recently, Zhang et al. used the $3^{r d}$ order tensors to represent the sNETs of a cohort of healthy individuals selected from the HCP dataset ${ }^{1}$ [25]. They decomposed it using tensor principal component analysis (TPCA) to show that several personal traits can be statistically significantly regressed using the 30-D representation of individual sNETs. Zhang's work improves over Durusoy's who had used a predefined basis subnetwork set, while imposing an orthogonality constraint on the identified basis subnetworks.

Building upon our previous work [24], we seek to identify the discriminative subnetworks using uni-modal (sNET / fNET) and multi-modal (sNET \& fNET) B-tensor factorizations for the diagnosis of the different stages of dementia (SCI - subjective cognitive impairment, MCI - mild cognitive impairment, AD - Alzheimer's Disease). These subnetworks' discriminative power is assessed using both multivariate ANOVA and classification performance in the low-dimensional space of subnetworks. The main contributions of this work are $(i)$ the first application of brain connectome tensor (B-tensor) factorization to neurodegenerative disease (specifically dementia) diagnostics, to the best of knowledge, (ii), demonstration of the high discriminative power of the extracted low-dimensional compact connectome spaces throughout the spectrum of dementia, (iii) structure-function fusion by means of multi-modal B-tensor factorization with parameter tying.

The rest of the paper is organized as follows: Section II describes the uni-modal and multi-modal B-tensor factorization, as well as the classifier training/testing scheme used for dementia diagnostics performance assessment. Section III provides a detailed description of the data cohort, the clinical assessment and reports the experimental results. Section IV discusses the results both from data science and neurology perspectives. Section V presents the concluding remarks and suggestions for future work.

\footnotetext{
${ }^{1}$ For each personal trait, two equal sized groups of individuals with maximal difference in their mean traits were selected. http://www.humanconnectomeproject.org/data/
}

\section{Methods}

A brain connectome is an undirected graph, $\mathcal{G}=(\mathcal{P}, \mathcal{E})$, where $\mathcal{P}(\#(\mathcal{P})=P)$ is the set of $P$ nodes/vertices corresponding to cortical brain regions/parcels and $\mathcal{E}$ is the set of undirected edges/associations between these regions. $\mathcal{G}$ is conventionally represented as a $P \times P$ symmetric matrix. The $3^{r d}$ and $4^{t h}$ order B-tensors are group-wide unified tensor representation of uni-modal and multi-modal brain connectomes, respectively. $\mathbf{B}_{f / s} \in \mathbb{R}_{+}^{P \times P \times N}$ is the $3^{r d}$ order non-negative uni-modal B-tensor of $P$-node connectomes for $N$ cases. We will use $\mathbf{B}_{f}$ and $\mathbf{B}_{s}$ for uni-modal functional (fNET) and structural (sNET) connectome B-tensors, respectively. The $4^{\text {th }}$ order B-tensor $\left(\mathbf{B}_{a} \in \mathbb{R}_{+}^{P \times P \times N \times M}\right)$ is the concatenation of $\mathbf{B}_{f}$ and $\mathbf{B}_{s}$ (i.e. $M=2$ ).

\section{A. Connectome Construction}

Each subject's T1w-MRI, fMRI, DWI volumes are coregistered and the T1w-MRI volumes are used to delineate the cortical regions by means of 148-parcel Destrieux atlas $(P=148)$ [26] registration, using a custom pre-processing pipeline $^{2}$ that utilizes the open source FreeSurfer, FSL and Tortoise toolboxes. Co-registered volumes are manually verified by neurologists prior to connectome construction. Identical parcellations (nodes) are used for all connectomes of an individual. In the rest, we will use the terms node and parcel interchangeably, as we do for connectivity and edge.

Diffusion tensor image (DTI) volumes are constructed from DWI volumes. The $4^{\text {th }}$-order Runge-Kutta (RK4) deterministic tractography algorithm is used with minimum fractional anisotropy (FA) set to 0.15 , stepsize set to $0.7 \mathrm{~mm}(\approx$ half the voxel size), minimum fiber length set to $20 \mathrm{~mm}$ and the maximum curvature set to $35^{\circ}$. RK4 was initiated from 30 randomly selected seeds per voxels with $F A>0.15$ [27]. In order to construct the sNETs, each fiber $\left(\left\{f_{k}\right\}\right)$ computed by RK4 is associated with the nodes in the vicinity of its endpoints through a normal weighting function. Let $W_{i k}$ represent the association between $i^{t h}$ parcel and $k^{t h}$ fiber defined as,

$$
W_{i k}=\sum_{\substack{\mathbf{r}_{j} \in P_{i} \\\left|\mathbf{r}_{j}-\mathbf{e}_{k}\right| \leq 2 \sigma}} \frac{1}{(2 \pi)^{3 / 2} \sigma^{3}} \exp \left(\frac{-\left|\mathbf{r}_{j}-\mathbf{e}_{k}\right|^{2}}{2 \sigma^{2}}\right)
$$

where $\mathbf{r}_{j}$ is the $j^{t h}$ voxel position in parcel $P_{i}, \mathbf{e}_{\mathbf{k}}$ is the $k^{t h}$ fiber's end-point (the closest one to $\mathbf{r}_{j}$ ). The $\sigma$ is predetermined, prior to connectome construction, as 0.155 following the methodology described in [28].

Three edge definitions are used for sNET construction, leading to three different sNETs with common nodes. The edge weights between nodes $P_{i}$ and $P_{j}$ are defined as,

$$
\begin{aligned}
e_{i j} & =\frac{2}{V_{i}+V_{j}} \sum_{k} W_{i k} W_{j k} \\
e_{i j}^{m} & =\frac{2}{V_{i}+V_{j}} \sum_{k}\left[W_{i k} W_{j k} \min _{s}\left(F A\left(f_{k}(s)\right)\right)\right] \\
e_{i j}^{a} & =\frac{2}{V_{i}+V_{j}} \sum_{k}\left[W_{i k} W_{j k} \operatorname{avg}_{s}\left(F A\left(f_{k}(s)\right)\right)\right]
\end{aligned}
$$

\footnotetext{
${ }^{2}$ https://vavlab.boun.edu.tr/research
} 
where $V_{i}$ and $V_{j}$ are the volumes of corresponding parcels in $m m^{3}, \min _{s}()$ and $\operatorname{avg}()$ are the minimum and the average operators over the arbitrarily parametrized (by $s$ ) fibers.

Two edge definitions are used for fNET construction, leading to two different fNETs with common nodes. More specifically, we used the absolute Pearson correlation coefficient (CC) and the absolute partial correlation coefficient (pCC) between the BOLD signals associated with each parcel to quantify functional associations [29]. The summary BOLD signals for each parcel are computed using the principal left singular vector of the singular value decomposition of the matrix of BOLD signals of all voxels in a given parcel, as described in [30].

\section{B. Uni-modal B-Tensor Factorization}

In order to find low dimensional connectome representations and orthogonal basis subnetworks, the following class balanced optimization problem is solved for the $3^{\text {rd }}$ order uni-modal B-tensors, $\mathbf{B}_{f}$ and $\mathbf{B}_{s}$, that represent fNETs and sNETs, respectively.

$$
\begin{aligned}
& \min _{d_{k}, v_{k}, u_{k}^{c}}\left\{\sum_{c=1}^{C} \frac{1}{N_{c}}\left\|\mathbf{B}_{f / s}^{c}-\sum_{k=1}^{K} d_{k}\left(v_{k} \circ v_{k} \circ u_{k}^{c}\right)\right\|_{2}^{2}\right\} \\
& \text { s.t. } \sum_{c=1}^{C}\left\|u_{k}^{c}\right\|_{2}^{2}=1 ; v_{k}^{T} v_{j}=\delta_{j k}
\end{aligned}
$$

where $C(=3$, for $\mathrm{SCI}, \mathrm{MCI}, \mathrm{AD})$ is the number of classes in the dataset, $N_{c}$ is the number of cases of class $c$, the free parameter $K$ is the number of subnetworks, i.e. the dimensionality of the connectome space. The subscripts referring to the modality that the B-tensor factors $\left(d_{k}, v_{k}, u_{k}^{c}\right)$ are associated with, are ignored to keep the notation simple. $d_{k} \in \mathbb{R}$ is the $k^{\text {th }}$ subnetwork's scaling factor, $\mathbf{V}_{k}=v_{k} \circ v_{k} \in \mathbb{R}^{P \times P}$ is the $k^{\text {th }}$ basis subnetwork and $u_{k}^{c} \in \mathbb{R}^{N_{c}}$ is the corresponding projection coefficient. Concatenating the K-dimensional representations of all $N$ cases, we get $\mathbf{U}_{f / s}=\left[u_{:}^{1} u_{:}^{2} u_{:}^{3}\right] \in \mathbb{R}^{K \times N}$. Equation 5 is blind to class labels of the cases, but uses individual B-tensors constructed for each class $\left(\mathbf{B}_{f / s}^{c}\right)$ with appropriate normalization in the optimization problem to overcome the class imbalance problem, rather than imposing equal class sizes by selecting cases from the cohort as is done in [25].

Following Allen, this single factor CP (canonical polyadic) decomposition can be rewritten as [31] ,

$$
\begin{aligned}
& \max _{v_{k}, u_{k}^{c}} \sum_{c=1}^{C} \frac{1}{N_{c}}\left(\mathbf{B}_{f / s}^{c} \times_{1}\left(\boldsymbol{\Gamma}_{k-1} v_{k}\right) \times_{2}\left(\boldsymbol{\Gamma}_{k-1} v_{k}\right) \times_{3} u_{k}^{c}\right) \\
& \text { s.t. } \sum_{c=1}^{C}\left\|u_{k}^{c}\right\|_{2}^{2}=1 ; v_{k}^{T} v_{j}=\delta_{j k}
\end{aligned}
$$

where $\boldsymbol{\Gamma}_{k-1}$ is the projection matrix onto the subspace orthogonal to the one spanned by $\left\{v_{1}, \cdots, v_{k-1}\right\}, \times_{n}$ denotes $\mathrm{n}$-mode tensor product [32]. The class balanced $3^{\text {rd }}$ order Btensor factorization algorithm is given in Algorithm 1, where $E_{\max }()$ is the principal eigenvector operator. We randomly initialized the B-tensor factorization 20 times and selected the one with minimum reconstruction error.

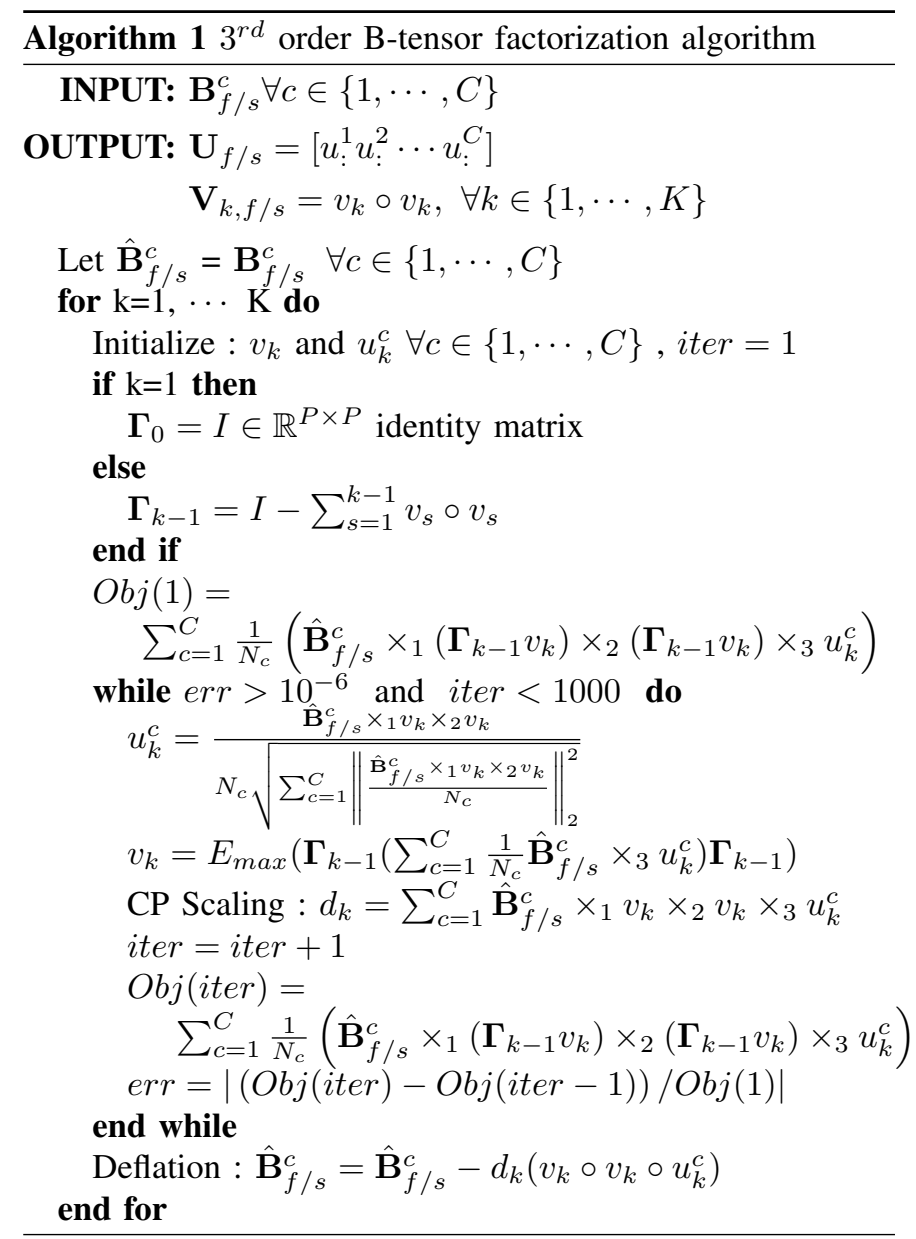

\section{Multi-modal B-tensor Factorization}

The $4^{\text {th }}$ order multi-modal B-tensor $\left(\mathbf{B}_{a}\right)$ is the concatenation of $\mathbf{B}_{f}$ and $\mathbf{B}_{s}$. Following an approach similar to the uni-modal B-tensor factorization, as described in Section II-B, the multi-modal $\mathbf{B}_{a}$ can be factorized, with the addition of a fourth factor, as,

$$
\begin{aligned}
& \min _{d_{k}, v_{k}, u_{k}^{c}, w_{k}}\left\{\sum_{c=1}^{C} \frac{1}{N_{c}}\left\|\mathbf{B}_{a}^{c}-\sum_{k=1}^{K} d_{k}\left(v_{k} \circ v_{k} \circ u_{k}^{c} \circ w_{k}\right)\right\|_{2}^{2}\right\} \\
& \text { s.t. } \sum_{c=1}^{C}\left\|u_{k}^{c}\right\|_{2}^{2}=1 ; v_{k}^{T} v_{j}=\delta_{j k} ; w_{k}^{T} w_{k} \leq 1
\end{aligned}
$$

where the additional factor $w_{k} \in \mathbb{R}^{2}$ is a scaling across the modalities (structure and function) for the $k^{t h}$ subnetwork, while all other factors and parameters are as described in Section II-B. Due to the difference in the sparsity of sNET and fNET connectivities, we have employed parameter tying and set $w_{k}=\left[w_{k}^{*} ; \alpha w_{k}^{*}\right]=\Theta w_{k}^{*}$, where $\alpha$ is a fixed density factor, defined as $\alpha=\operatorname{density}(\mathrm{fNET}) / \operatorname{density}(\mathrm{sNET})$, and $w_{k}^{*}$ is the tying parameter.

Following Allen again, the above minimization problem can 


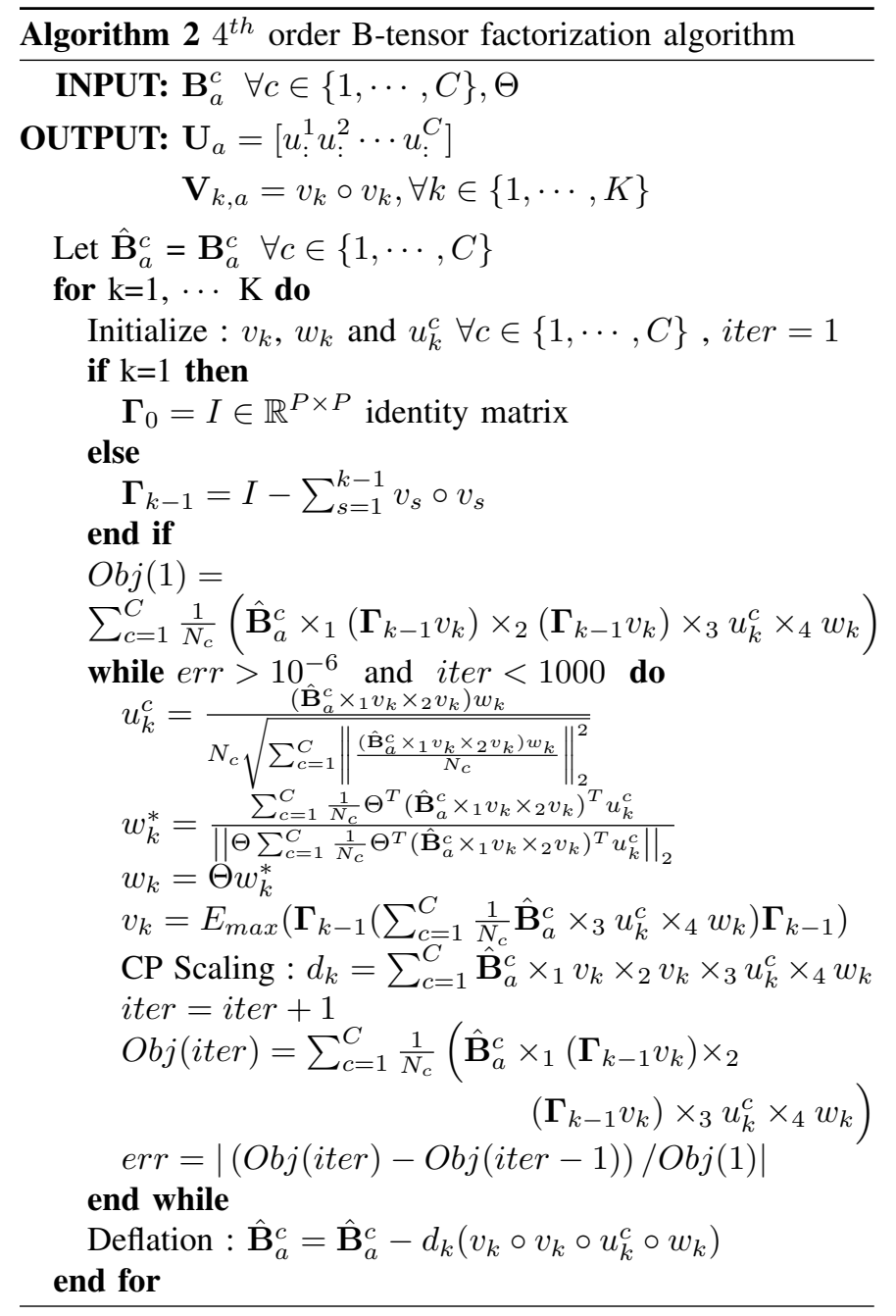

be posed as a maximization problem [31],

$\max _{v_{k}, u_{k}^{c}, w_{k}} \sum_{c=1}^{C} \frac{1}{N_{c}}\left(\mathbf{B}_{a}^{c} \times_{1}\left(\boldsymbol{\Gamma}_{k-1} v_{k}\right) \times_{2}\left(\boldsymbol{\Gamma}_{k-1} v_{k}\right) \times_{3} u_{k}^{c} \times_{4} w_{k}\right)$

s.t. $\sum_{c=1}^{C}\left\|u_{k}^{c}\right\|_{2}^{2}=1 ; v_{k}^{T} v_{j}=\delta_{j k} ; w_{k}^{T} w_{k} \leq 1$

where $\boldsymbol{\Gamma}_{k-1}$ is the projection matrix as before. We get the class balanced $4^{\text {th }}$ order B-tensor factorization as in Algorithm 2 where $E_{\max }()$ is the principal eigenvector operator, $\Theta=\left[\begin{array}{ll}1 & \alpha\end{array}\right]^{T}$ with $\alpha$ being a fixed sparsity ratio between the structural and functional connectomes. Derivations of $u_{k}^{c}, v_{k}$ and $w_{k}^{*}$ are given in Appendix A. As before, we randomly initialized the B-tensor factorization 20 times and selected the one with minimum reconstruction error.

\section{Classification in Low Dimensional Connectome Space}

The B-tensor factorizations explained in Sections II-B and II-C allow us to represent each individual connectome in a $\mathrm{K}$ D space with columns of $\mathbf{U}_{f}, \mathbf{U}_{s}$ and $\mathbf{U}_{a}$, corresponding to fNETs, sNETs and multi-modal structure-function representation. These K-dimensional spaces are spanned by basis subnetworks denoted by $\mathbf{V}_{k, f}, \mathbf{V}_{k, s}$ and $\mathbf{V}_{k, a}$. Let $\mathbf{u}_{f / s / a}(n) \in \mathbb{R}^{K}$ denote the $n^{\text {th }}$ case's K-D connectome representation. The significance of class separation in K-D spaces is assessed by multivariate ANOVA [33].

We adopted a leave-one-out cross-validation scheme to evaluate the performance of a 3-class (SCI/MCI/AD) linear SVM classifier $^{3}$ on K-D vectorial representations $\left(u_{\text {: }}\right)$ of cases. The classification accuracies and confusion matrices are reported in Tables II and III. The statistical significance of the observed classification performances is assessed using the Fisher-Pitman permutation test [34], [35] for which we randomly shuffled the class labels of subjects 1000 times without altering the K-D connectome representations and followed the same leave-oneout cross-validation training/testing scheme.

\section{EXPERIMENTS}

\section{A. Data and Clinical Assessment}

88 volunteers were enrolled in the study with written consent and under an ethics committee approval ${ }^{4}$. We used an age and gender matched subset of 47 individuals (17 male, 30 female, age $=61.4 \pm 5.6$ ). There were three diagnostic groups: Early-stage AD dementia (AD) $\left(N_{1}=8\right)$, mild cognitive impairment of the amnestic type (MCI) $\left(N_{2}=25\right)$, and subjective cognitive impairment $(\mathrm{SCI})\left(N_{3}=14\right)$. Table I summarizes the information about subjects with respect to amnestic type. The participants underwent routine clinical examination at Istanbul University, Istanbul Faculty of Medicine, Department of Neurology, Behavioral Neurology and Movement Disorders Unit and their MR scans were acquired in a single session using the Philips Achieva 3T MRI system (Best, Netherlands) with a 32-channel head coil at Neuroimaging Unit of Hulusi Behçet Life Sciences Research Laboratory, Istanbul University. We used 3D FFE (Fast Field Echo) pulse sequence with multi-shot TFE (Turbo Field Echo) imaging mode for T1-weighted MRI. The acquisition parameters were $\mathrm{TE} / \mathrm{TR}=3.8 \mathrm{~ms} / 8.3 \mathrm{~ms}$, flip-angle $=8^{\circ}$, SENSE reduction 2 (Foot-Head), FOV $=220(R L) \times 240(A P) \mathrm{mm}^{2}$, voxel size $=$ $1.0 \times 1.0 \times 1.0 \mathrm{~mm}^{3}$ and number of slices $=180$. DWI were acquired with a maximum gradient strength of $40 \mathrm{mT} / \mathrm{m}$, and $200 \mathrm{mT} / \mathrm{m} / \mathrm{ms}$ slew rate, using a single-shot, pulse-gradient spin echo (PGSE), echo planar imaging (EPI) sequence. The acquisition parameters were $\mathrm{FOV}=200 \times 236 \mathrm{~mm}^{2}, 2.27 \mathrm{~mm}$ isotropic voxel size, $112 \times 112$ reconstruction matrix, 71 slices and $T E / T R=92 \mathrm{~ms} / 9032 \mathrm{~ms} .120$ diffusion weighting gradient directions were used at various b-values between $0-3000 \mathrm{~s} / \mathrm{mm}^{2}$.

The diagnosis of amnestic type MCI was made based on NIA-AA criteria [36]. An objective deficit in episodic memory was defined by a total free recall score (TFR) of 27 or lower and Cue Index (CI) of 0.67 or lower on the Free and Cued Selective Reminding Test (FCSRT) [37]. Thus, all the nondemented participants who scored above 27 in TFR-FCSRT and above 0.67 in CI-FCSRT were automatically labeled as

\footnotetext{
${ }^{3}$ One-vs-one multiclass SVM classifier is trained and used. We used Matlab $^{\mathrm{TM}}$ 's multiclass model fitting function fitcecoc with its default parameters except Prior, which is set to Uniform.

${ }^{4}$ Istanbul University, Istanbul Faculty of Medicine, Ethics Committee Approval: 877/30.05.2014 ; Bogazici University, Ethics Committee Approval: 2014-1/17.02.2014
} 
TABLE I

SUMMARY OF AGE AND GENDER MATCHED DATASET.

\begin{tabular}{|l|c|c|c|}
\hline Type & AD & MCI & SCI \\
\hline \# of Female/Male & $5 / 3(62.5 \% \mathrm{~F})$ & $16 / 9(64 \% \mathrm{~F})$ & $9 / 5(64 \% \mathrm{~F})$ \\
\hline Age $\in[51,70]$ & $62.25 \pm 6.61$ & $61.28 \pm 5.31$ & $61.00 \pm 5.99$ \\
\hline CI-FCSRT & $0.31 \pm 0.21$ & $0.66 \pm 0.17$ & $0.87 \pm 0.12$ \\
\hline TFR-FCSRT & $7.25 \pm 8.31$ & $21.92 \pm 4.54$ & $34.5 \pm 3.94$ \\
\hline
\end{tabular}

TABLE II

LEAVE-ONE-OUT CROSS-VALIDATED CLASSIFICATION ACCURACIES FOR K-DIMENSIONAL BRAIN CONNECTOME REPRESENTATIONS. THE P-VALUES OF THE MULTIVARIATE ANOVA TESTS FOR EACH REPRESENTATION ARE ALL LESS THAN $1 e-09$.

\begin{tabular}{|c|c|c|c|c|c|c|c|}
\hline \multirow{2}{*}{} & \multicolumn{7}{|c|}{ Number of Basis Subnetworks (K) } \\
\cline { 2 - 8 } & 3 & 5 & 7 & 9 & 11 & 13 & 15 \\
\hline \multicolumn{7}{|c|}{ Functional Uni-modal $\left(\mathbf{U}_{f}\right)$} \\
\hline CC & 0.96 & 1.00 & 1.00 & 1.00 & 0.96 & 0.94 & 0.94 \\
pCC & 1.00 & 0.96 & 0.98 & 0.94 & 0.96 & 0.96 & 0.94 \\
\hline \multicolumn{7}{|c|}{ Structural Uni-modal $\left(\mathbf{U}_{s}\right)$} \\
\hline NW & 0.85 & 0.91 & 0.87 & 0.85 & 0.85 & 0.83 & 0.83 \\
mFA & 0.87 & 0.81 & 0.79 & 0.77 & 0.79 & 0.85 & 0.85 \\
aFA & 0.81 & 0.79 & 0.81 & 0.79 & 0.81 & 0.87 & 0.87 \\
\hline Multi-modal $\left(\mathbf{U}_{a}\right)$ & 1.00 & 1.00 & 1.00 & 1.00 & 1.00 & 0.98 & 0.98 \\
NW+pCC & 1.00 & 0.98 & 0.94 & 1.00 & 0.96 \\
mFA+pCC & 1.00 & 1.00 & 0.98 & 0.98 & 0.96 & 0.96 & 0.96 \\
aFA+pCC & 1.00 & 1.00 & 0.98 & 0.98 & 0.96 & 1.00 & 0.98 \\
NW+CC & 1.00 & 1.00 & 1.00 & 1.00 & 1.00 & 1.00 \\
mFA+CC & 0.96 & 1.00 & 1.00 & 1.00 & 0.96 & 0.94 & 0.96 \\
aFA+CC & 0.96 & 0.98 & 1.00 & 1.00 & 0.98 & 1.00 & 0.98 \\
\hline
\end{tabular}

SCI. This score of 27 was chosen as a more liberal cutoff, compared to the 24 , which was shown to be a highly sensitive measure of predicting future dementia in dementiafree individuals [38]. AD participants, on the other hand, were selected among those who fulfill NIA-AA criteria for probable $\mathrm{AD}$ with an amnestic presentation [39]. SCI subjects were volunteers responding to the calls of the project and they scored at least 1 on either Cognitive Functions Instrument Subject form (CFI-S) or CFI Study Partner form (CFI-SP) [40]. After a comprehensive neurological and neuropsychological examination and cranial magnetic resonance imaging (MRI), the diagnoses of SCI, MCI, and AD were confirmed by a panel of behavioral neurologists. The panel also ensured that all AD participants had a Clinical Dementia Rating Scale (CDR) score of 0.5 or 1 , thus had a very mild or mild dementia. All MCI subjects had a CDR score of 0.5 and CDR Sum-ofBoxes (CDR-SOB) score of 0.5 or 1 . All SCI subjects had a CDR score of 0 . Participants with a history of current or past neurological or psychiatric disorders adversely affecting cognition, alcohol or substance abuse, major head trauma with loss of consciousness, white matter hyperintensities on MRI with a Fazekas score of 2 and 3, as well as contraindications for scanning at MRI were other exclusion criteria from our study.

\section{B. Results}

We ran experiments with two types of fNETs constructed using absolute Pearson correlation coefficient (CC) and absolute partial correlation coefficient (pCC) as connectivity definitions, and three types of sNETs constructed using the structural connectivity definitions given in Equations 2, 3, 4, namely the normalized weighted fiber count (NW), minimum fractional anisotropy (mFA) and average fractional anisotropy (aFA) based connectivity definitions, respectively. For all options, we used $K \in\{3,5,7,9,11,13,15\}$. All mean B-tensor reconstruction errors among the 20 random initializations were less than $1 e-4$ for all experiments.

Table II shows the leave-one-out cross-validation classification accuracies for uni-modal and multi-modal B-tensor factors $\mathbf{u}_{f / s}$ and $\mathbf{u}_{a}$, respectively, for all sNET and fNET definitions. The statistical significance of the inter-group (SCI/MCI/AD) separation for uni-modal and multi-modal B-tensor factors is reasserted by the multivariate ANOVA tests for all cases. The classification accuracies show that the uni-modal fNET factorizations $\left(\mathbf{u}_{f}\right)$ generally perform better than the unimodal sNET $\left(\mathbf{u}_{s}\right)$ factorizations. The added value of multi-modal factorization is evident in all cases, though the differences in classification accuracy are small when compared with unimodal fNET factorizations. An overall assessment of Table II suggests $K=5$ as a reasonable choice in terms of classification accuracy and low dimensionality. The null hypothesis (that all three classes have equal means) was rejected by the multivariate ANOVA test with a p-value less that $1 e-09$ in all cases, indicating that $\mathrm{AD}, \mathrm{MCI}$, and $\mathrm{SCI}$ subjects fall into easily distinguishable clusters in the low-dimensional connectome spaces. We assessed the statistical significance of the reported classification performances of the trained classifiers by means of the Fisher-Pitman permutation test. To this end, we randomly shuffled the class labels of subjects $N_{s h f}(=1000)$ times without altering their K-D connectome representations. We then followed the same leave-one-out cross-validations training/testing scheme to get samples from the null distribution of accuracies. All entries in Table II were found to be extreme outliers, since none of the label permutations returned a larger accuracy. Corresponding $\mathrm{p}$ values are therefore less than $1 e-03\left(=1 / N_{s h f}\right)$, consistent with the multivariate ANOVA test.

Table III shows the confusion matrices for the classifiers trained on uni-modal B-tensor factorizations for $K=5$ and 15. The majority of the misclassifications are observed with structural connectomes and between AD-SCI groups. Among all, the fractional anisotropy (FA) based sNETs perform worse, which is parallel with previously reported results in literature albeit for different tasks [25].

Figure 1 summarizes the distribution of projection coefficients $\left(u_{f / s / a}\right)$ per subnetwork $(K=5)$ and per group (SCI/MCI/AD). The CP scaling factors $\left(d_{k}\right.$ 's) are measures of variation expressed by the corresponding subnetwork and are reported as percentages. An immediate observation is that most of the fNET variation is confined to a single subnetwork, which alone is highly discriminative of SCI/MCI/AD groups, as is evident from the distribution of $u_{f}$ 's for that specific subnetwork. The sNET variation is more complex and distributed relatively evenly across all 5 subnetworks. The intra-group variation is relatively higher in $\mathrm{AD}$ group, followed by the SCI group, which can partially explain the higher confusion between $\mathrm{AD}$ and SCI reported in Table 
TABLE III

CONFUSION MATRICES FOR 3-CLASS CLASSIFICATION WITH $K=5$ AND $K=15$ FACTORIZATIONS OF UNIMODAL B-TENSORS

\begin{tabular}{|c|c|c|c|c|c|c|c|}
\hline & \multicolumn{3}{|c|}{$\mathrm{K}=5$} & \multicolumn{3}{|c|}{$\mathrm{K}=15$} \\
\hline & & \multicolumn{6}{|c|}{ Predicted } \\
\hline & & $\mathrm{SCI}$ & MCI & $\mathrm{AD}$ & SCI & MCI & $\mathrm{AD}$ \\
\hline \multirow{3}{*}{ u } & SCI & 14 & 0 & 0 & 14 & 0 & 0 \\
\hline & MCI & 0 & 25 & 0 & 0 & 25 & 0 \\
\hline & $\mathrm{AD}$ & 0 & 0 & 8 & 2 & 1 & 5 \\
\hline \multirow{3}{*}{ U. } & SCI & 14 & 0 & 0 & 14 & $\overline{0}$ & $\overline{0}$ \\
\hline & MCI & 0 & 25 & 0 & 0 & 25 & 0 \\
\hline & $\mathrm{AD}$ & 2 & 0 & 6 & 3 & 0 & 5 \\
\hline \multirow{3}{*}{ z } & SCI & 12 & 1 & 1 & 11 & 2 & 1 \\
\hline & MCI & 1 & 24 & 0 & 3 & 22 & 0 \\
\hline & $\mathrm{AD}$ & 1 & 0 & 7 & 2 & 0 & 6 \\
\hline \multirow{3}{*}{ 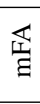 } & SCI & 11 & 2 & 1 & 12 & 0 & 2 \\
\hline & MCI & 2 & 23 & 0 & 1 & 24 & 0 \\
\hline & $\mathrm{AD}$ & 4 & 0 & 4 & 4 & 0 & 4 \\
\hline \multirow{3}{*}{ 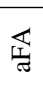 } & SCI & 9 & 4 & 1 & 11 & 2 & 1 \\
\hline & MCI & 3 & 22 & 0 & 2 & 23 & 0 \\
\hline & $\mathrm{AD}$ & 2 & 0 & 6 & 1 & 0 & 7 \\
\hline
\end{tabular}

III. The multi-modal B-tensor factorization demonstrates a dimensionality similar to sNETs', while having a better group separation than both uni-modal factorization in general. The results suggest certain functional and structural subnetworks to be highly discriminative.

The B-tensor factorization further offers an insight into the structural and functional mechanism(s) that manifest dementia, beyond offering a highly accurate diagnostics. The brain's subnetworks were already suggested as biomarkers of $\mathrm{AD}$ [41]. Figure 2 depicts the basis subnetworks that we have identified via uni-modal and multi-modal B-tensor factorizations. A preliminary neurological assessment of the structural subnetworks reveals the precuneus, the angular gyrus, the supramarginal gyrus, the superior parietal lobule, the precentral gyrus and the postcentral gyrus as the most conspicuous regions. The precuneus is probably the single most salient structure within the neuropathological process of $\mathrm{AD}$ as the first structure that is involved by the initial amyloid load during the pre-clinical stages of the amyloid accumulation. This accumulation disrupts both the intra and inter-network connectivity even during the pre-clinical stage [42], [43]. Even the genetically susceptible individuals without amyloid load were shown to exhibit disrupted precuneus connectivity [44]. According to the Braak\&Braak staging, the angular gyrus, the supramarginal gyrus and the superior parietal lobule are involved in stage $\mathrm{V}$ of neurofibrillary tangles spread, reflecting the earliest phase of dementia [45]. The precentral and postcentral gyri are the most unexpected findings. They harbor primary motor and somatosensory cortices and are not thought to be involved in the neuropathological spread, even at the final stage VI of Braak\&Braak [45]. Unlike sNETs, the CP scaling factors of fNETs are rather uneven, indicating the first subnetwork as, by far, the most significant one. The precuneus and dorsal \& ventral parts of posterior cingulate cortex (PCC) are functionally the most conspicuous cortical regions. They are connected to various bilateral hubs, with distinct functional and structural properties, and their contralateral mirror reflections. The left precuneus is specifically strongly expressed in the most significant subnetwork, with asymmetrical connections. As described above, the precuneus is a key region for dementia. The dorsal \& ventral parts of PCC are specifically the components of the Papez circuit and thus thought to be affected at the Braak\&Braak stage III-IV. Consequently, the preliminary neurological assessment confirms that the identified structural and functional connectivities agree with neurological literature, while suggesting some unexpected connectivities which deserve further studies with regard to their role in the disease spectrum. No direct relation between the B-tensor basis subnetworks and the seven functional subnetworks was observed [23]. These seven subnetworks are depicted with color codes in Figure 2.

\section{Discussion}

Comparing the uni-modal and the multi-modal B-tensor factorization results in Table II, the higher performance of fNETs over sNETs is clearly observed. This is in agreement with the previously reported results, which form the bulk of the current literature on connectome analysis for neurodegenerative disease [46]. However, we also observed a rather high performance with sNETs, which is a strong evidence that dementia manifests also on the structure of the brain networks [47]. Further, the multi-modal B-tensor factorization approach improves the accuracy beyond unimodal analysis. The multi-modal B-tensor factorization offers a new perspective to brain connectomes where an underlying fundamental network structure is suggested, of which fNETs and sNETs are independent observations. This is in line with recent findings which suggest that different MRI modalities provide complementary information that helps to discriminate AD groups [48]. The multi-modal B-tensor factorization offers a novel and effective method to fuse functional and structural information in connectome analysis.

$\beta$-amyloid $(\mathrm{A} \beta)$ peptide and $\mathrm{p}$-tau concentrations in cerebro-spinal fluid (CSF) were observed to be related to different stages of Alzheimer's disease (AD) [49], [50]. The A $\beta$ concentration decreases in pre-clinical stages and strongly corresponds to the $\mathrm{A} \beta$ neuropathology and the diagnosis of $\mathrm{AD}$ (continuum), while the p-tau concentration increases along the pre-dementia and dementia stages of $\mathrm{AD}$ and correlates with clinical disease severity. A subset of 35 subjects from our cohort had their CSF tests available. Figure 3 depicts the distribution of $\mathrm{p}$-tau and $\mathrm{A} \beta$ for this subset and per group (SCI/MCI/AD). While $\mathrm{A} \beta$ marks the $\mathrm{AD}$ group clearly, it is incapable of discriminating clinically identifiable SCI and MCI groups. On the other hand, p-tau concentration follows the expected trend, yet falls short of clearly separating the clinical groups. However, the proposed B-tensor factorization showed high separability throughout the disease spectrum, and is potentially a highly sensitive and non-invasive clinical biomarker. These preliminary observations also suggest that discriminative changes in brain connectomes exist even in the absence of significant changes in $\mathrm{A} \beta$ and p-tau levels.

The dataset size is a major limitation for drawing conclusive results with regard to clinical applicability. Our results justify further studies with a larger and preferably multi-center dataset 

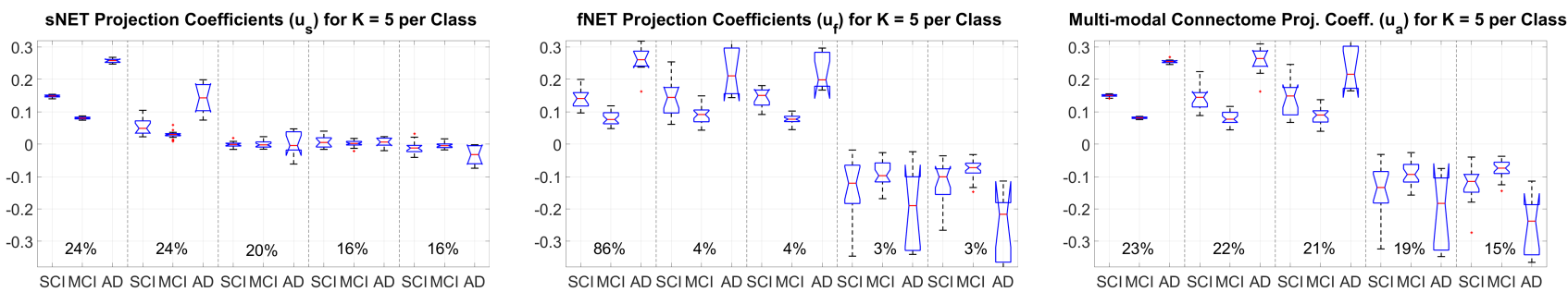

Fig. 1. The distribution of projection coefficients (left-to-right: $u_{s}: \mathrm{NW}, u_{f}: \mathrm{pCC}, u_{a}: \mathrm{NW}+\mathrm{pCC}$ ) per subnetwork (left-to-right: $1^{\text {st }}-5^{\text {th }}$ ) and per group (SCI/MCI/AD). The CP scaling factors $\left(d_{k}\right.$ 's) for each subnetwork is a measure of the variation explained by the corresponding subnetwork and is given as a percentage for each subnetwork.
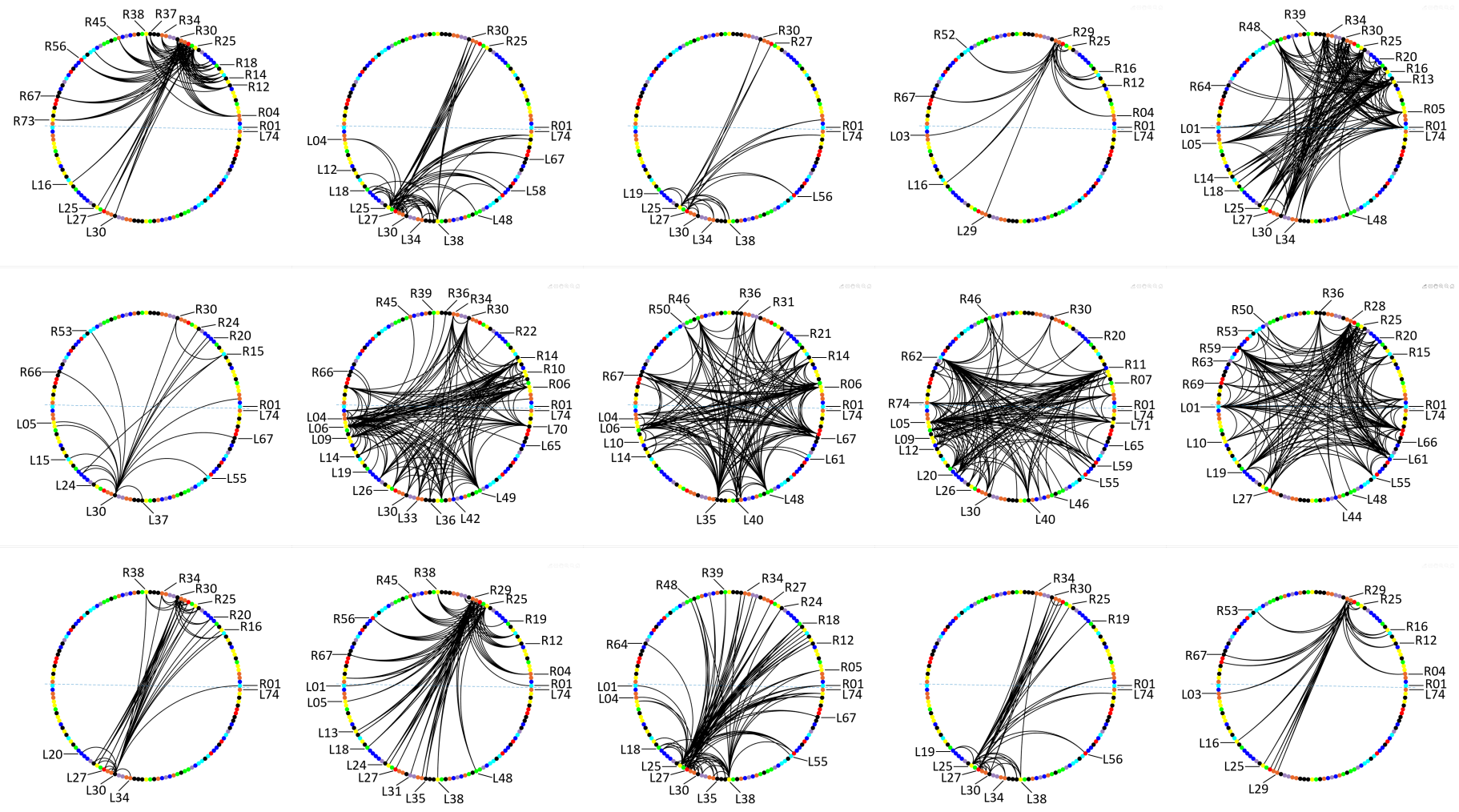

Fig. 2. The basis subnetworks, in order of significance (left-to-right), extracted by the unimodal structural (upper row), functional (middle row) and the multi-modal (lower row) B-tensor factorization for $K=5$. The 148 nodes of the Destrieux atlas [26] are color coded following the seven functional networks (Red: Dorsal Attention, Green: Salience, Blue: Visual , Cyan: Frontoparietal, Purple: Limbic, Orange: Somatomotor \& Auditory , Yellow: Default Mode, Black: Multiple Overlapping Networks) described in literature [23]. The significantly strong connections $(>\mu+3.5 \sigma)$ are displayed for presentation purposes. The precuneus (L/R30), the angular gyrus (L/R25), the supramarginal gyrus (L/R26), the superior parietal lobule (L/R27), the precentral gyrus (L/R29), the postcentral gyrus (L/R28), the dorsal \& ventral parts of posterior cingulate cortex (L/R 9-10) are observed to be the most conspicuous regions.

which would not only increase the statistical power but also shed light on the effect of data acquisition variations, which will be important in a clinical setting. A study with followup cases, over the course of full disease development, may prove to be beneficial in identifying AD-susceptible SCI cases which would have a much higher impact than diagnosis. An important and novel contribution of B-tensor factorization is the insight it offers towards understanding the disease development. The current neurological assessment of the identified subnetworks generally agree with current knowledge, however, we have also made controversial observations such as the involvement of the precentral and the postcentral gyri. Such observations deserve further assessment. The B-tensor factorization algorithm is based on the CP factorization recently employed by Zhang et al. [25]. It is an intuitive approach to project a connectome onto a minimum dimensional space, so much as a PCA approach. Nevertheless, tensor factorization is an optimization problem and an active research area in data analytics [51]-[53]. A comparative assessment of different tensor factorization methods applied to B-tensor factorization would be a valuable contribution, which is beyond our scope and is left for future research. 


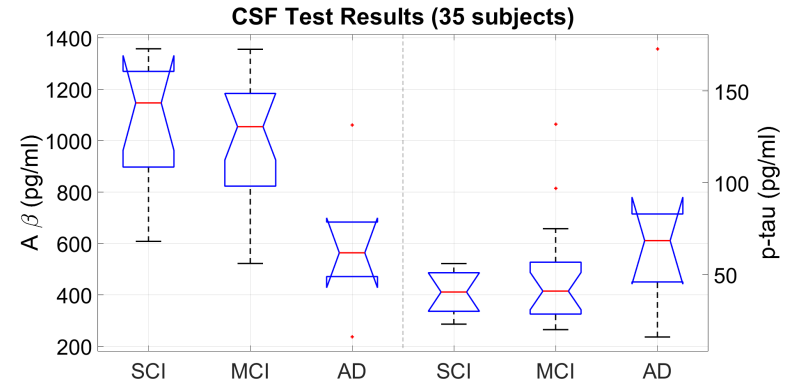

Fig. 3. The $\mathrm{A} \beta$ and $\mathrm{p}$-tau concentrations of 35 subjects that have undergone $\mathrm{CSF}$ tests. $\mathrm{A} \beta$ decreases and $\mathrm{p}$-tau increases for $\mathrm{AD}$ cases, while the MCI and the SCI groups are not well-separated.

\section{CONCLUSION}

Multi-dimensional tensor factorization offers a unique and powerful methodology to explore the underlying connectivity patterns (subnetworks) in brain connectomes, as well as an efficient way to fuse multi-modal data. The unimodal class balanced B-tensor factorization suggests a low dimensional highly discriminative structural connectome space with highly and relatively equally significant basis subnetworks. In parallel with literature, the micro-structure (fractional anisotropy) dependent connectivity definitions did not perform better than simpler weighted tract counts. The identified discriminative structural basis subnetworks mostly agree with the neurological knowledge, yet also suggest unexpected discriminative connections, such as the pre- and post-central gyri. These findings need to be further assessed from a neurological point of view. Unlike the structural connectomes, a single functional basis subnetwork singles out with high discriminative power throughout the disease continuum. The multi-modal B-tensor factorization, in general, had better classification performance, with lower intra-class variation, suggesting the efficient fusion of structure and function. For all cases, MCI group fell further apart from SCIs and ADs, which could be attributed to the expected higher variation, that the B-tensor factorization captures, in MCI stage which is a transition stage. Further studies are needed to assess the relation between these low dimensional connectome representations and multiple clinical findings, as well as the significance of the connections that are unexpectedly observed to be part of discriminative subnetworks. A blind study is also due to assess the discriminative power of the projections of connectomes onto the basis subnetworks identified in this study.

\section{APPENDIX A \\ Balanced Multi-Modal B-Tensor Factorization With PARAMETER TYING}

Let $X^{c}$ be the $4^{\text {th }}$ order semi-symmetric tensor. $X^{c} \in$ $\mathbb{R}_{+}^{P \times P \times N_{c} \times M}$ of $P \times P$ connectomes of $N_{c}$ cases of class $c(c \in\{1, \cdots, C\})$ for $M$ modalities. The multi-modal B- tensor factorization is posed as an optimization problem [31],

$$
\begin{aligned}
& \max _{v, u^{c}, w} \sum_{c=1}^{C} \frac{1}{N_{c}}\left(X^{c} \times_{1} v \times_{2} v \times_{3} u^{c} \times_{4} w\right) \\
& \text { s.t. } \sum_{c=1}^{C}\left\|u^{c}\right\|_{2}^{2}=1 ; v^{T} v=1 ; w^{T} w \leq 1
\end{aligned}
$$

where $v \in \mathbb{R}^{P}, u^{c} \in \mathbb{R}^{N_{c}}$ and $w \in \mathbb{R}^{M}$. Using Lagrange multipliers,

$$
\begin{aligned}
& L\left(v, u^{c}, w\right)=\sum_{c=1}^{C} \frac{1}{N_{c}} X^{c} \times_{1} v \times_{2} v \times_{3} u^{c} \times_{4} w \\
& -\lambda_{1}\left(\sum_{c=1}^{C}\left(u^{c}\right)^{T}\left(u^{c}\right)-1\right)-\lambda_{2}\left(v^{T} v-1\right)-\lambda_{3}\left(w^{T} w-1\right)
\end{aligned}
$$

Taking the partial derivative w.r.t. $u^{c}$,

$$
\begin{array}{r}
\frac{\partial L}{\partial u^{c}}=\frac{1}{N_{c}}\left(\left(X^{c} \times_{1} v \times_{2} v\right) w\right)^{T}-2 \lambda_{1}\left(u^{c}\right)^{T}=0 \\
\Rightarrow u^{c}=\frac{\left(X^{c} \times_{1} v \times_{2} v\right) w}{N_{c} 2 \lambda_{1}} \\
\lambda_{1}\left(\sum_{c=1}^{C}\left(u^{c}\right)^{T}\left(u^{c}\right)-1\right)=0 \Rightarrow \sum_{c=1}^{C}\left\|u^{c}\right\|_{2}^{2}=1 \\
\Rightarrow \sum_{c=1}^{C}\left\|\frac{X^{c} \times_{1} v \times_{2} v}{N_{c} 2 \lambda_{1}}\right\|_{2}^{2}=1 \\
\Rightarrow \lambda_{1}=\frac{1}{2} \sqrt{\sum_{c=1}^{C}\left\|\frac{\left(X^{c} \times_{1} v \times_{2} v\right) w}{N_{c}}\right\|_{2}^{2}}
\end{array}
$$

Equations 12 and 13 give,

$$
u^{c}=\frac{\left(X^{c} \times_{1} v \times_{2} v\right) w}{N_{c} \sqrt{\sum_{c=1}^{C}\left\|\frac{\left(X^{c} \times_{1} v \times_{2} v\right) w}{N_{c}}\right\|_{2}^{2}}}
$$

Taking the derivative of Equation 10 w.r.t. $v$,

$$
\begin{array}{r}
\frac{\partial L}{\partial v}=2 \sum_{c=1}^{C} \frac{1}{N_{c}} v^{T}\left(X^{c} \times_{3} u^{c} \times_{4} w\right)-2 \lambda_{2} v^{T}=0 \\
\Rightarrow \sum_{c=1}^{C} \frac{1}{N_{c}}\left(X^{c} \times_{3} u^{c} \times_{4} w\right)^{T} v=\lambda_{2} v
\end{array}
$$

we get an eigenvalue decomposition problem where $\left(\lambda_{2}, v\right)$ is an eigenvalue-eigenvector pair. Maximum eigenvalue and the corresponding eigenvector maximizes Equation $9{ }^{5}$. Let $E_{\max }($.$) denote the eigenvector corresponding to the maxi-$ mum eigenvalue, then we get $v$ as

$$
v=E_{\max }\left(\sum_{c=1}^{C} \frac{1}{N_{c}}\left(X^{c} \times_{3} u^{c} \times_{4} w\right)^{T}\right)
$$

\footnotetext{
${ }^{5}$ Equation 9 can be rewritten as $\max _{v, u^{c}, w} \sum_{c=1}^{C} \frac{1}{N_{c}} v^{T}\left(X^{c} \times{ }_{3} u^{c} \times{ }_{4} w\right)^{T} v$
} 
Finally, letting $w=\left[\begin{array}{l}1 \\ \alpha\end{array}\right] w^{*}=\Theta w^{*}$, where $\alpha$ is the sparsity ratio of the two connectome modalities, $w^{*} \in \mathbb{R}^{2 \times 1}$, and taking the derivative of Equation 10 w.r.t. $w^{*}$,

$$
\begin{array}{r}
\frac{\partial L}{\partial w^{*}}=\sum_{c=1}^{C} \frac{1}{N_{c}}\left(u^{c}\right)^{T}\left(X^{c} \times_{1} v \times_{2} v\right) \Theta \\
-2 \lambda_{3}\left(w^{*}\right)^{T} \Theta^{T} \Theta=0 \\
w^{*}=\frac{\sum_{c=1}^{C} \frac{1}{N_{c}} \Theta^{T}\left(X^{c} \times_{1} v \times_{2} v\right)^{T} u^{c}}{2 \lambda_{3} \Theta^{T} \Theta}
\end{array}
$$

and $\lambda_{3}$ can be found as :

$$
\begin{aligned}
\sum_{c=1}^{C}\left\|\Theta w^{*}\right\|_{2}^{2} & =\left\|\frac{\Theta \sum_{c=1}^{C} \frac{1}{N_{c}} \Theta^{T}\left(X^{c} \times_{1} v \times_{2} v\right)^{T} u^{c}}{2 \lambda_{3} \Theta^{T} \Theta}\right\|_{2}^{2}=1 \\
\Rightarrow \lambda_{3} & =\frac{1}{2}\left\|\frac{\Theta \sum_{c=1}^{C} \frac{1}{N_{c}} \Theta^{T}\left(X^{c} \times_{1} v \times_{2} v\right)^{T} u^{c}}{\Theta^{T} \Theta}\right\|_{2}
\end{aligned}
$$

Equations 19 and 20 give,

$$
w^{*}=\frac{\sum_{c=1}^{C} \frac{1}{N_{c}} \Theta^{T}\left(X^{c} \times_{1} v \times_{2} v\right)^{T} u^{c}}{\left\|\Theta \sum_{c=1}^{C} \frac{1}{N_{c}} \Theta^{T}\left(X^{c} \times_{1} v \times_{2} v\right)^{T} u^{c}\right\|_{2}}
$$

from which we have $w=\left[\begin{array}{l}1 \\ \alpha\end{array}\right] w^{*}$

\section{ACKNOWLEDGMENT}

This work was in part supported by the Turkish Directorate of Strategy and Budget under the TAM Project number 2007K12-873, TÜBİTAK-ARDEB 1003 Programme under Grant \#114E053, Bogazici University Research Fund Grant \#16862. We would specifically like to thank Dr. A. Taylan Cemgil for his valuable discussions, to Abdullah Karaaslanlı, Zeynep Kahraman and Dr. M. Okan İrfanoğlu for their contributions to preprocessing.

\section{REFERENCES}

[1] "World population ageing," 2019. [Online]. Available: https://www.un.org/en/development/desa/population/publications/ pdf/ageing/WorldPopulationAgeing2019-Highlights.pdf

[2] C. Reitz, C. Brayne, and R. Mayeux, "Epidemiology of Alzheimer disease," Nature reviews Neurology, vol. 5, no. 6, pp. 137-152, 2011.

[3] C. Reitz and R. Mayeux, "Alzheimer disease: epidemiology, diagnostic criteria, risk factors and biomarkers," Biochemical pharmacology, vol. 88 , no. 4 , pp. $640-651,2014$.

[4] M. Wortmann, "Importance of national plans for Alzheimer's disease and dementia," Alzheimer's research \& therapy, vol. 5, no. 5, p. 40, 2013.

[5] X. Delbeuck, M. Van der Linden, and F. Collette, "Alzheimer's disease as a disconnection syndrome?" Neuropsychology review, vol. 13 , no. 2 , pp. 79-92, 2003.

[6] J. J. Palop, J. Chin, and L. Mucke, "A network dysfunction perspective on neurodegenerative diseases," Nature, vol. 443, no. 7113, p. 768, 2006.

[7] R. C. Craddock, S. Jbabdi, C.-G. Yan, J. T. Vogelstein, F. X. Castellanos, A. Di Martino, C. Kelly, K. Heberlein, S. Colcombe, and M. P. Milham, "Imaging human connectomes at the macroscale," Nature methods, vol. 10, no. 6, p. 524, 2013.

[8] H.-J. Park and K. Friston, "Structural and functional brain networks: from connections to cognition," Science, vol. 342, no. 6158, p. 1238411 , 2013.

[9] A. Fornito, A. Zalesky, and M. Breakspear, "Graph analysis of the human connectome: promise, progress, and pitfalls," Neuroimage, vol. 80, pp. 426-444, 2013.
[10] D. S. Bassett and E. T. Bullmore, "Human brain networks in health and disease," Current opinion in neurology, vol. 22, no. 4, p. 340, 2009.

[11] B. M. Tijms, A. M. Wink, W. de Haan, W. M. van der Flier, C. J. Stam, P. Scheltens, and F. Barkhof, "Alzheimer's disease: connecting findings from graph theoretical studies of brain networks," Neurobiology of aging, vol. 34, no. 8, pp. 2023-2036, 2013.

[12] A. Fornito, A. Zalesky, and E. Bullmore, Fundamentals of brain network analysis. Academic Press, 2016.

[13] E. J. Sanz-Arigita, M. M. Schoonheim, J. S. Damoiseaux, S. A Rombouts, E. Maris, F. Barkhof, P. Scheltens, and C. J. Stam, "Loss of small-worldnetworks in alzheimer's disease: graph analysis of fmri resting-state functional connectivity," PloS one, vol. 5, no. 11, p. e13788, 2010.

[14] C. J. Stam, B. Jones, G. Nolte, M. Breakspear, and P. Scheltens, "Smallworld networks and functional connectivity in Alzheimer's disease," Cerebral cortex, vol. 17, no. 1, pp. 92-99, 2006.

[15] K. Supekar, V. Menon, D. Rubin, M. Musen, and M. D. Greicius, "Network analysis of intrinsic functional brain connectivity in Alzheimer's disease," PLoS computational biology, vol. 4, no. 6, 2008.

[16] M. Daianu, N. Jahanshad, T. M. Nir, A. W. Toga, C. R. Jack Jr, M. W. Weiner, and P. M. Thompson, for the Alzheimer's Disease Neuroimaging Initiative, "Breakdown of brain connectivity between normal aging and Alzheimer's disease: a structural k-core network analysis," Brain connectivity, vol. 3, no. 4, pp. 407-422, 2013.

[17] M. Daianu, N. Jahanshad, T. M. Nir, C. R. Jack Jr, M. W. Weiner, M. A. Bernstein, P. M. Thompson, and A. D. N. Initiative, "Rich club analysis in the Alzheimer's disease connectome reveals a relatively undisturbed structural core network," Human brain mapping, vol. 36, no. 8, pp. 30873103, 2015.

[18] A. Dragomir, A. G. Vrahatis, and A. Bezerianos, "A network-based perspective in Alzheimer's disease: Current state and an integrative framework," IEEE journal of biomedical and health informatics, vol. 23, no. 1 , pp. $14-25,2018$

[19] A. Zalesky, A. Fornito, and E. T. Bullmore, "Network-based statistic: identifying differences in brain networks," Neuroimage, vol. 53, no. 4, pp. 1197-1207, 2010.

[20] J. Kim, W. Pan, A. D. N. Initiative et al., "Highly adaptive tests for group differences in brain functional connectivity," Neuroimage: Clinical, vol. 9, pp. 625-639, 2015.

[21] H. Eavani, T. D. Satterthwaite, R. Filipovych, R. E. Gur, R. C. Gur, and C. Davatzikos, "Identifying sparse connectivity patterns in the brain using resting-state fMRI," Neuroimage, vol. 105, pp. 286-299, 2015.

[22] P. Zille, V. D. Calhoun, J. M. Stephen, T. W. Wilson, and Y.-P. Wang, "Fused estimation of sparse connectivity patterns from rest fMRI: Application to comparison of children and adult brains," IEEE transactions on medical imaging, vol. 37, no. 10, pp. 2165-2175, 2017.

[23] B. Thomas Yeo, F. M. Krienen, J. Sepulcre, M. R. Sabuncu, D. Lashkari, M. Hollinshead, J. L. Roffman, J. W. Smoller, L. Zöllei, J. R. Polimeni et al., "The organization of the human cerebral cortex estimated by intrinsic functional connectivity," Journal of neurophysiology, vol. 106, no. 3, pp. 1125-1165, 2011.

[24] G. Durusoy, A. Karaaslanlı, D. Y. Dal, Z. Yıldırım, and B. Acar, "Multimodal brain tensor factorization: Preliminary results with AD patients," in International Workshop on Connectomics in Neuroimaging. Springer, 2018, pp. 29-37.

[25] Z. Zhang, G. I. Allen, H. Zhu, and D. Dunson, "Tensor network factorizations: Relationships between brain structural connectomes and traits," Neuroimage, vol. 197, pp. 330-343, 2019.

[26] C. Destrieux, B. Fischl, A. Dale, and E. Halgren, "Automatic parcellation of human cortical gyri and sulci using standard anatomical nomenclature," Neuroimage, vol. 53, no. 1, pp. 1-15, 2010.

[27] C. Tench, P. Morgan, M. Wilson, and L. Blumhardt, "White matter mapping using diffusion tensor MRI," Magnetic Resonance in Medicine, vol. 47 , no. 5 , pp. 967-972, 2002.

[28] D. Moyer, B. A. Gutman, J. Faskowitz, N. Jahanshad, and P. M. Thompson, "Continuous representations of brain connectivity using spatial point processes," Medical image analysis, vol. 41, pp. 32-39, 2017.

[29] G. Marrelec, A. Krainik, H. Duffau, M. Pélégrini-Issac, S. Lehéricy, J. Doyon, and H. Benali, "Partial correlation for functional brain interactivity investigation in functional MRI," Neuroimage, vol. 32, no. 1, pp. 228-237, 2006.

[30] F. D. Bowman, L. Zhang, G. Derado, and S. Chen, "Determining functional connectivity using fMRI data with diffusion-based anatomical weighting," Neuroimage, vol. 62, no. 3, pp. 1769-1779, 2012.

[31] G. Allen, "Sparse higher-order principal components analysis," in Artificial Intelligence and Statistics, 2012, pp. 27-36. 
[32] T. Kolda and B. Bader, "Tensor decompositions and applications," SIAM review, vol. 51, no. 3, pp. 455-500, 2009.

[33] R. Johnson and D. Wichern, Applied Multivariate Statistical Analysis, ser. Pearson Modern Classics for Advanced Statistics Series. Pearson, 2018.

[34] P. Good, Permutation tests: a practical guide to resampling methods for testing hypotheses. Springer Science \& Business Media, 2013.

[35] R. J. Boik, "The fisher-pitman permutation test: A non-robust alternative to the normal theory f test when variances are heterogeneous," British Journal of Mathematical and Statistical Psychology, vol. 40, no. 1, pp. 26-42, 1987.

[36] M. S. Albert, S. T. DeKosky, D. Dickson, B. Dubois, H. H. Feldman, N. C. Fox, A. Gamst, D. M. Holtzman, W. J. Jagust, R. C. Petersen et al., "The diagnosis of mild cognitive impairment due to Alzheimer's disease: recommendations from the national institute on aging-Alzheimer's association workgroups on diagnostic guidelines for Alzheimer's disease," Alzheimer's \& dementia, vol. 7, no. 3, pp. 270-279, 2011.

[37] H. Buschke, "Selective reminding for analysis of memory and learning," Journal of Verbal Learning and Verbal Behavior, vol. 12, no. 5, pp. 543$550,1973$.

[38] E. Grober, A. E. Sanders, C. Hall, and R. B. Lipton, "Free and cued selective reminding identifies very mild dementia in primary care," Alzheimer disease and associated disorders, vol. 24, no. 3, p. 284, 2010

[39] G. M. McKhann, D. S. Knopman, H. Chertkow, B. T. Hyman, C. R. Jack Jr, C. H. Kawas, W. E. Klunk, W. J. Koroshetz, J. J. Manly, R. Mayeux et al., "The diagnosis of dementia due to Alzheimer's disease: recommendations from the national institute on aging-Alzheimer's association workgroups on diagnostic guidelines for Alzheimer's disease," Alzheimer's \& dementia, vol. 7, no. 3, pp. 263-269, 2011.

[40] C. Li, J. Neugroschl, X. Luo, C. Zhu, P. Aisen, S. Ferris, and M. Sano, "The utility of the cognitive function instrument (CFI) to detect cognitive decline in non-demented older adults," Journal of Alzheimer's Disease, vol. 60, no. 2, pp. 427-437, 2017.

[41] S. Teipel, M. J. Grothe, J. Zhou, J. Sepulcre, M. Dyrba, C. Sorg, and C. Babiloni, "Measuring cortical connectivity in Alzheimer's disease as a brain neural network pathology: toward clinical applications," Journal of the International Neuropsychological Society, vol. 22, no. 2, pp. 138163, 2016.

[42] V. L. Villemagne, K. E. Pike, G. Chetelat, K. A. Ellis, R. S. Mulligan, P. Bourgeat, U. Ackermann, G. Jones, C. Szoeke, O. Salvado, R. Martins, G. O'Keefe, C. Mathis, W. Klunk, D. Ames, C. Masters, and C. Rowe, "Longitudinal assessment of $\mathrm{A} \beta$ and cognition in aging and Alzheimer disease," Ann Neurol, vol. 69, no. 1, pp. 181-192, 2011.

[43] Y. I. Sheline, M. E. Raichle, A. Z. Snyder, J. C. Morris, D. Head, S. Wang, and M. A. Mintun, "Amyloid plaques disrupt resting state default mode network connectivity in cognitively normal elderly," Biol Psychiatry, vol. 67, no. 6, pp. 584-587, 2010.

[44] Y. I. Sheline, J. C. Morris, A. Z. Snyder, J. L. Price, Z. Yan, G. D'Angelo, L. Collin, D. Sachin, B. Tammie, F. Anne, G. Alison, and M. A. Mintun, "APOE4 allele disrupts resting state fMRI connectivity in the absence of amyloid plaques or decreased CSF Abeta42," J Neurosci, vol. 30, no. 50, pp. $17035-17040,2010$.

[45] M. Mesulam, Principles of behavioral and cognitive neurology. Oxford University Press, 2000.

[46] C. Hohenfeld, C. J. Werner, and K. Reetz, "Resting-state connectivity in neurodegenerative disorders: Is there potential for an imaging biomarker?" Neuroimage: Clinical, vol. 18, pp. 849-870, 2018.

[47] L. R. Peraza, A. Díaz-Parra, O. Kennion, D. Moratal, J.-P. Taylor, M. Kaiser, R. Bauer, A. D. N. Initiative et al., "Structural connectivity centrality changes mark the path toward Alzheimer's disease," Alzheimer's \& Dementia: Diagnosis, Assessment \& Disease Monitoring, vol. 11, pp. 98-107, 2019.

[48] T. M. Schouten, M. Koini, F. de Vos, S. Seiler, J. van der Grond, A. Lechner, A. Hafkemeijer, C. Möller, R. Schmidt, M. de Rooij et al., "Combining anatomical, diffusion, and resting state functional magnetic resonance imaging for individual classification of mild and moderate Alzheimer's disease," Neuroimage: Clinical, vol. 11, pp. 46-51, 2016.

[49] C. Jack, D. S. Knopman, W. J. Jagust, L. Shaw, P. Aisen, M. Weiner, R. Petersen, and J. Trojanowski, "Hypothetical model of dynamic biomarkers of the Alzheimer's pathological cascade," The Lancet Neurology, vol. 9, no. 1, pp. 119-128, 2010.

[50] C. Jack, D. Bennett, K. Blennow, M. Carrillo, B. Dunn, H. S.B., D. Holtzman, W. Jagust, J. Jessen, F. amd Karlawish, E. Liu, J. Molinuevo, T. Montine, C. Phelps, K. Rankin, C. Rowe, P. Scheltens, E. Siemers, H. Snyder, and R. Sperling, "NIA-AA research framework: Toward a biological definition of Alzheimer's disease." Alzheimers Dement, vol. 14, no. 4, pp. 535-562, 2018.
[51] M. Che, A. Cichocki, and Y. Wei, "Neural networks for computing best rank-one approximations of tensors and its applications," Neurocomputing, vol. 267, pp. 114-133, 2017.

[52] M. Che and Y. Wei, "Randomized algorithms for the approximations of tucker and the tensor train decompositions," Adv. Comput. Math. vol. 45, pp. 395-428, 2019.

[53] M. Che, Y. Wei, and H. Yan, "The computation of low multilinear rank approximations of tensors via power scheme and random projection," SIAM J. Matrix Anal. Appl., vol. 41, pp. 605-636, 2020. 